\title{
Changements climatiques et maladies infectieuses: À quoi pouvons-nous nous attendre?
}

\author{
NH Ogden ${ }^{1,2 *}$, P Gachon ${ }^{3}$
}

\section{Résumé}

Les changements climatiques à l'échelle mondiale, causés par les émissions de gaz à effet de serre d'origine humaine, ont une incidence particulière au Canada, où le réchauffement est en général plus élevé que dans le reste du monde. Le réchauffement continu sera accompagné de changements dans les précipitations, qui varieront selon les régions et les saisons, et d'une variabilité accrue du climat et de phénomènes météorologiques extrêmes. Les changements climatiques entraîneront probablement l'émergence de maladies infectieuses au Canada, qui se propageront vers le nord en provenance des États-Unis ou seront introduites par le transport maritime et aérien international. Des maladies endémiques au Canada pourraient aussi réapparaître.

Ce numéro spécial traite des principaux risques de maladies infectieuses associés aux changements climatiques. II s'agit entre autres de l'émergence des maladies transmises par des tiques, outre la maladie de Lyme, de la possible introduction de maladies exotiques transmises par des moustiques, comme la malaria et la dengue, d'un plus grand nombre d'épidémies de maladies vectorielles endémiques au Canada, comme le virus du Nil occidental, et une incidence accrue des maladies transmises par des virus d'origine alimentaire. Le risque devrait être aggravé par le fait qu'une population vieillissante souffrant de maladies chroniques a une plus grande sensibilité aux maladies infectieuses. Cerner les risques de maladies émergentes afin d'évaluer notre vulnérabilité est un processus essentiel et constitue un point de départ pour établir où doivent porter les efforts en santé publique pour réduire la vulnérabilité et l'exposition de la population canadienne.

Cette oeuvre est mise à la disposition selon les termes de la licence internationale Creative Commons Attribution 4.0

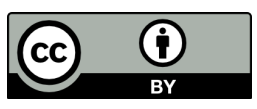

Affiliations

${ }^{1}$ Division des sciences des risques pour la santé publique, Laboratoire national de microbiologie, Agence de la santé publique du Canada, SaintHyacinthe (Québec)

${ }^{2}$ Division des sciences des risques pour la santé publique, Laboratoire national de microbiologie, Agence de la santé publique du Canada, Guelph (Ontario)

${ }^{3}$ Centre pour l'étude et la simulation du climat à l'échelle régionale (ESCER), Université du Québec à Montréal, Montréal (Québec)

Citation proposée : Ogden NH, Gachon P. Changements climatiques et maladies infectieuses : À quoi pouvonsnous nous attendre? Relevé des maladies transmissibles au Canada 2019;45(4):83-8.

https://doi.org/10.14745/ccdr.v45i04a01f

Mots clés : changement climatique, maladie chronique, maladie de Lyme, maladie transmise par des moustiques, maladie à transmission vectorielle, origine alimentaire, précipitation, température
*Correspondance:

nicholas.ogden@canada.ca

\section{Introduction}

Les articles contenus dans ce numéro du Relevé des maladies transmissibles au Canada présentent un aperçu de la façon dont les changements climatiques peuvent augmenter le nombre et l'étendue des maladies à transmission vectorielle et l'incidence des infections d'origine alimentaire au Canada (1-4). Cet éditorial résume les changements climatiques, récents et à venir, au Canada; la façon dont les changements climatiques pourraient influencer l'émergence et la réémergence de maladies infectieuses; et la façon dont, à la lumière de l'évolution démographique et de l'état de santé des Canadiens, ces changements pourraient influer sur les risques de maladies infectieuses.

\section{Changements climatiques, récents et à venir, au Canada}

Les tendances au réchauffement se sont accélérées à l'échelle mondiale, alors que la température annuelle de l'air dans le monde a augmenté de près de $1{ }^{\circ} \mathrm{C}$ de 1880 à 2017 (5). Les années 2015 à 2017 ont nettement été plus chaudes que toutes les années antérieures (6), et les trois dernières décennies l'ont été davantage que toutes les décennies précédentes depuis 1850 (7). Cette tendance varie géographiquement. Ainsi, le réchauffement des bassins arctiques et subarctiques, particulièrement dans le nord-est du Canada, est plus élevé et plus rapide, en raison de la diminution accélérée de la couverture de neige et de glace de mer $(8,9)$. 
Depuis 1948, le taux de réchauffement au Canada dans son ensemble a été deux fois supérieur à la moyenne mondiale, et le taux de réchauffement dans le nord du Canada (nord du 60e parallèle nord) a été en gros au moins trois fois supérieur à la moyenne mondiale (10). Dans le nord-est du Canada (nord du 60 e parallèle nord et est du 110e méridien ouest), la température moyenne annuelle a augmenté de 0,75 à $1,2^{\circ} \mathrm{C}$ par décennie au cours des trois dernières décennies, comparativement à environ $0,18{ }^{\circ} \mathrm{C}$ par décennie à l'échelle mondiale (5). La température moyenne de l'air continuera d'augmenter en fonction de l'augmentation des concentrations de gaz à effet de serre dans l'atmosphère découlant des activités humaines.

D'ici les années 2070, la température de la majorité du territoire canadien devrait être de $5{ }^{\circ} \mathrm{C}$ plus élevée que la température de la période de référence 1971 à 2000 (Figure 1a). Les changements prévus concernant les précipitations totales annuelles incluent de légères augmentations dans les provinces des Prairies et des augmentations plus élevées (principalement sous forme de pluie) dans le nord et l'est du Canada. Toutefois, les projections relatives aux précipitations sont moins fiables qu'elles ne le sont pour la température (Figure 1b).

Dans le futur, le réchauffement devrait être plus élevé dans le nord-est du Canada (une augmentation allant jusqu'à $8,65^{\circ} \mathrm{C}$ d'ici les années 2070) (Figure 2), en raison de la diminution importante de la couverture de neige et de glace de mer d'ici la fin du 21e siècle (13).
Les changements de températures et de précipitations à long terme devraient être accompagnés d'une variabilité accrue des températures et de la pluie d'une année à l'autre ainsi que de phénomènes météorologiques extrêmes, y compris des vagues de chaleur et des pluies intenses augmentant les risques d'inondations (13). Une importante augmentation de I'intensité annuelle moyenne des précipitations par jour de pluie (jusqu'à $15 \%$ d'ici les années 2070), mais avec une diminution du nombre de jours de pluie par année, est prévue dans le nord-est du Canada, où le réchauffement sera plus prononcé au cours du 21 e siècle. Il est prévu que l'intensité des précipitations augmente considérablement au fil du temps en raison du taux de réchauffement, ce qui touchera aussi les régions du sud et de l'est du Canada

\section{La façon dont les changements climatiques peuvent influer sur l'émergence et la réémergence des maladies infectieuses}

\begin{abstract}
Au cours des dix dernières années, nous avons vu l'émergence et la réémergence de maladies infectieuses partout dans le monde, y compris la maladie à virus Ebola en Afrique, le coronavirus du syndrome respiratoire du Moyen-Orient (CoV-SRMO) au MoyenOrient, ainsi que la maladie à virus Zika, le chikungunya, la fièvre jaune et la dengue dans les Amériques. Ces maladies posent d'énormes défis en matière de santé publique.
\end{abstract}

Figure 1 : Changements prévus a) de la température annuelle moyenne (en ${ }^{\circ} \mathrm{C}$ ) et b) des précipitations totales annuelles (en \%) pour la période de 2071 à 2100 comparativement à la période de 1971 à 2000
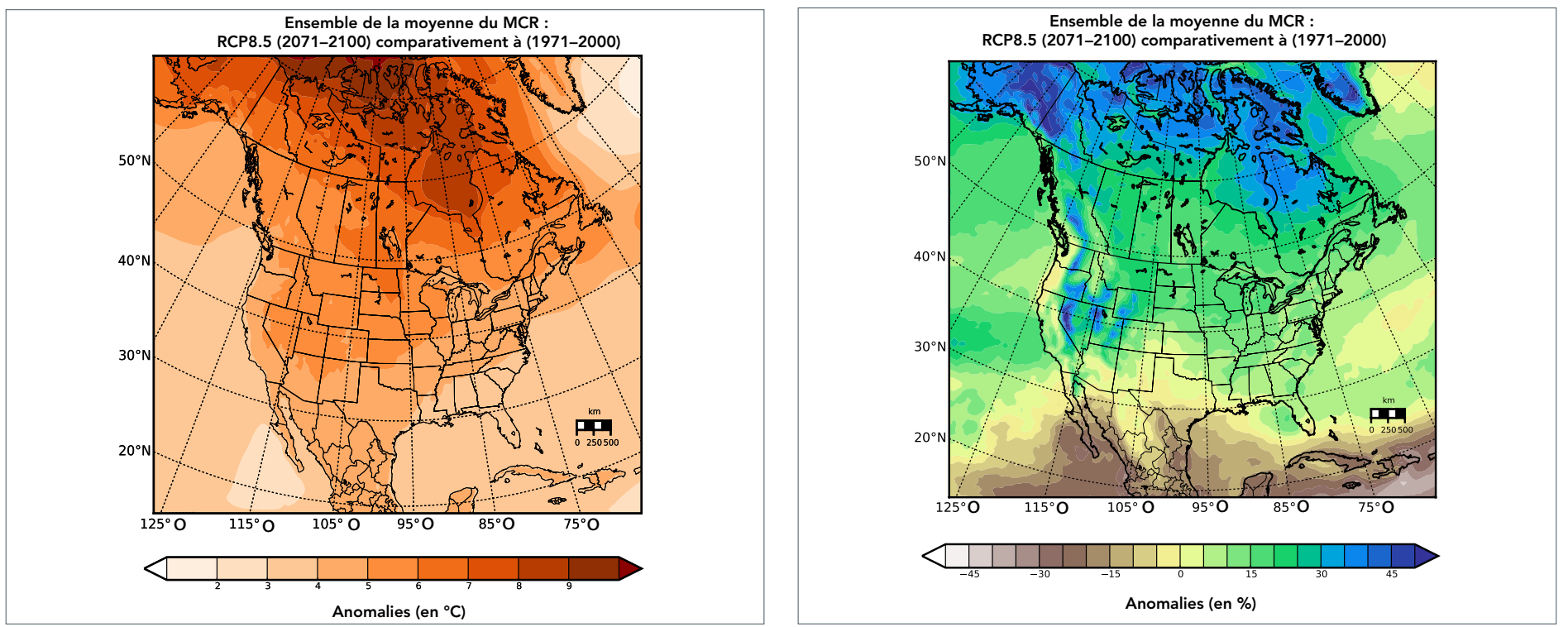

Abréviations: $M C R$, modèle climatique régional; $R C P$, profil représentatif d'évolution de concentration

NB: Simulations à l'aide de neuf modèles climatiques régionaux (MCR) et des ensembles de données disponibles provenant du projet CORDEX (COordinated Regional climate Downscaling

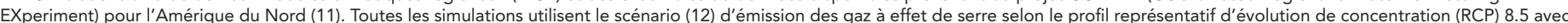

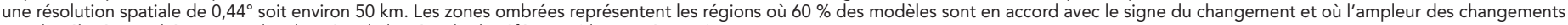
est plus élevée que l'écart type des données de la période de référence (de 1971 à 2000) 
Figure 2 : Variations annuelles observées et prévues des températures moyennes quotidiennes dans le nordest du Canada de 1948 à 2100 comparativement à la période de 1971 à 2000

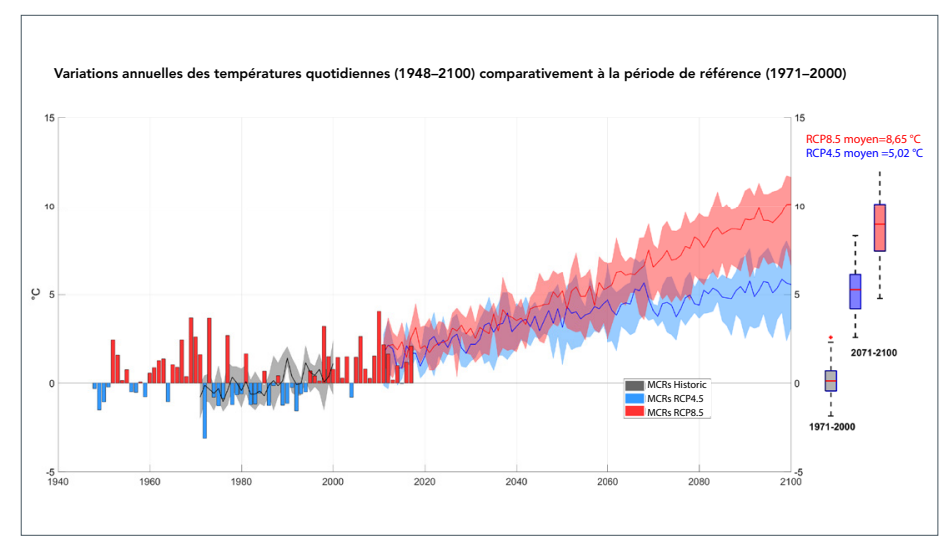

Abréviations: $M C R$, modèle climatique régional; $R C P$, profil représentatif d'évolution de concentration

a Le nord-est du Canada désigne les territoires situés au nord du 60e parallèle nord et à l'est du 110 e méridien ouest

NB : Les variations annuelles de la température moyenne quotidienne figurent sur les histogrammes pour les données observées de référence et sur les lignes pour les variations simulées. Les changements prévus reposent sur les mêmes simulations de modèle utilisées pour la Figure 1 à l'aide de deux scénarios d'émission RCP4.5 (ligne bleue) et RCP8.5 (ligne rouge) ce dernier étant pour le moment le plus réaliste. Les ombres entourant les lignes be rouge, ce dé et rouge (médiane de toutes les simulations) montrent l'étendue de l'augmentation prévue des températures selon les différents modèles climatiques régionaux. À droite, les diagrammes de quartiles montrent la médiane et la gamme des températures (variation interquartile) pour la période de référence (c.-à-d. les données observées) et pour la période de 2071 à 2100 (c.-à-d. les projections des modèles) selon les deux différents RCP (12)

Des maladies infectieuses apparaissent en raison de modifications de leur répartition géographique et par "émergence adaptative ", un changement génétique touchant les microorganismes qui infectent des animaux (généralement des animaux sauvages) de telle sorte que ces microorganismes peuvent infecter les êtres humains et que la transmission devient peut-être possible entre les êtres humains (14) - en d'autres mots, il s'agit d'une adaptation génétique qui produit une nouvelle maladie zoonotique.

L'émergence d'une maladie a de multiples facteurs, qui sont entre autres associés aux changements environnements ( $y$ compris les changements climatiques), aux changements sociaux et démographiques (y compris la mondialisation), et aux modifications apportées aux politiques et aux systèmes de santé publique (15). Les mêmes facteurs peuvent favoriser la réémergence de maladies endémiques (c.-à-d. l'augmentation d'une incidence ou une réapparition sous forme d'épidémies). Le climat et les changements climatiques peuvent avoir des effets directs sur l'émergence et la réémergence de maladies infectieuses en influant sur la survie des agents pathogènes, la survie et la reproduction des vecteurs arthropodes, la contamination de l'eau et, dans le cas des zoonoses, l'abondance d'hôtes réservoirs (c.-à-d. les animaux porteurs de microbes). Ces effets directs des changements climatiques sur l'écologie et la transmission aux êtres humains d'agents infectieux ont déjà fait l'objet d'évaluations à l'échelle nationale $(16,17)$ et mondiale (18-20). Toutefois, les changements climatiques peuvent avoir des effets indirects sur l'émergence et la réémergence de maladies, en influant sur d'autres changements sociaux et environnementaux ainsi que sur les systèmes de santé publique.

Les répercussions des changements climatiques sur les écosystèmes, y compris les effets sur la biodiversité, peuvent modifier le risque d'une nouvelle maladie zoonotique transmise par un animal sauvage $(21,22)$. De plus, les changements climatiques peuvent avoir un effet défavorable sur l'économie mondiale, particulièrement dans les pays à faible et moyen revenu. Que ce soit directement ou en augmentant la fréquence des conflits, un tel effet pourrait réduire la surveillance des maladies infectieuses et contribuer à élever la densité des agents infectieux dans les pays à l'extérieur de l'Amérique du Nord. Des effets défavorables sur l'économie des pays à faible et moyen revenu peuvent entraîner l'augmentation du nombre de réfugiés ou de migrants économiques et, ainsi, la propagation accrue de maladies infectieuses au Canada (23).

Les effets combinés de tous ces facteurs sont la source de trois importantes répercussions prévues des changements climatiques :

- Les risques accrus de l'introduction et de la transmission endémique de maladies infectieuses " exotiques » (à la fois directement transmissible et à transmission vectorielle) en provenance de partout dans le monde (comme le syndrome respiratoire aigu sévère [SRAS])

- La propagation du sud vers le nord de maladies actuellement endémiques aux États-Unis (comme I'anaplasmose)

- La réémergence (c.-à-d. un changement dans les comportements et les aires de répartition favorisant les épidémies) de maladies infectieuses endémiques au Canada (comme les éclosions du virus du Nil occidental) (Figure 3)

Les changements à long terme dans les températures et les précipitations, la variabilité accrue du climat, et la fréquence plus élevée des phénomènes météorologiques extrêmes, décrits ci-dessus, influeront sur les différents risques de maladies infectieuses de manière particulière (24). De plus en plus, des études de modélisation sont élaborées pour aider à prévoir les effets des changements climatiques sur les maladies infectieuses, dans le but de nous permettre de mieux nous préparer à faire face à ces risques changeants.

\section{Autres facteurs à considérer}

Au Canada, l'évolution des tendances relatives aux maladies infectieuses associées aux changements climatiques doit aussi être prise en considération dans le contexte d'autres tendances relatives aux maladies associées aux changements démographiques et à la santé des Canadiens.

Puisque la population canadienne vieillit et est de plus en plus atteinte de maladies chroniques, il faudra examiner ensemble les risques de maladies infectieuses et les risques de 
Figure 3 : Un résumé de l'incidence des changements climatiques sur les risques de maladies infectieuses au Canada

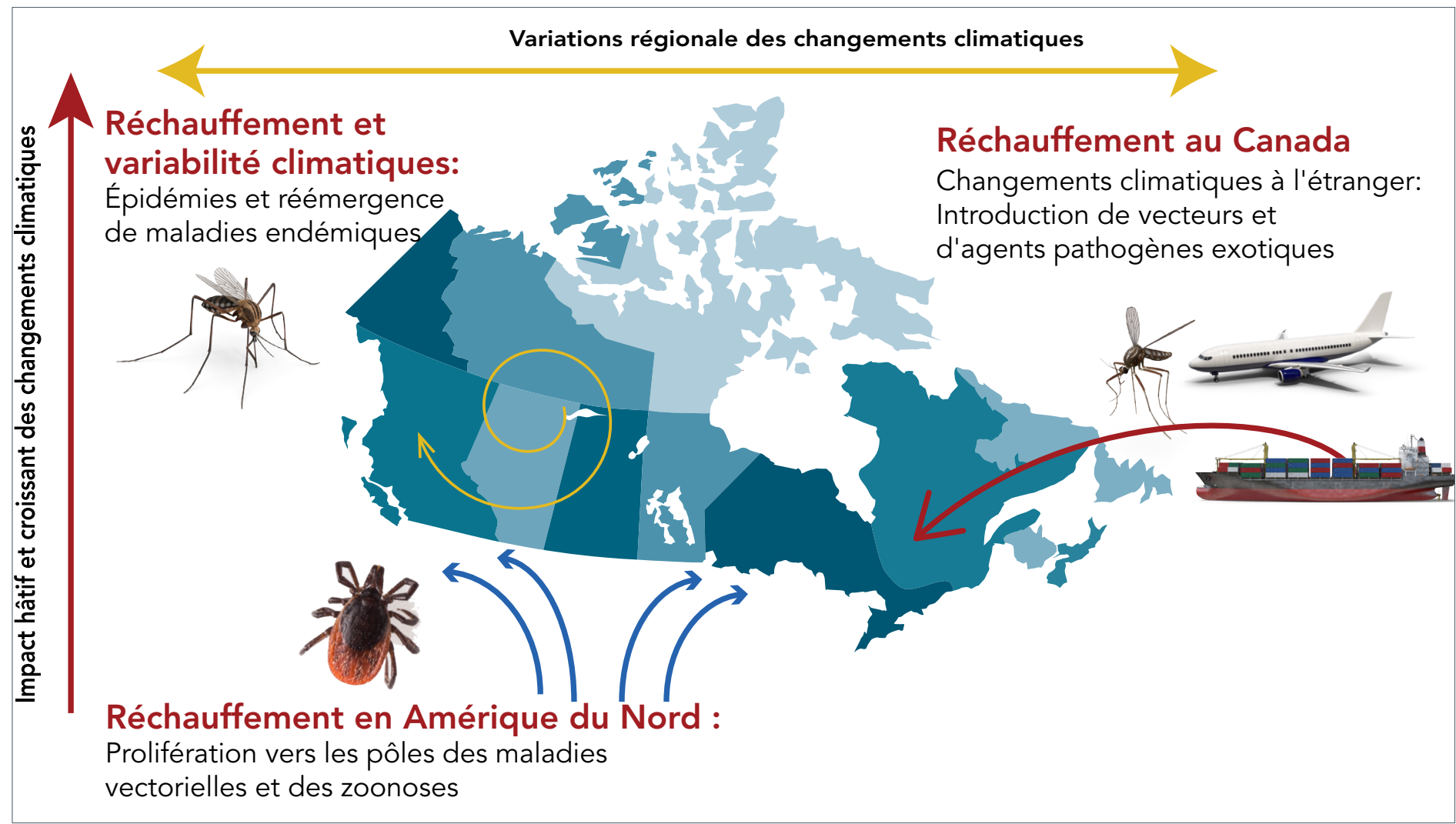

Modifié (23)

maladies chroniques (25). Les risques de maladies infectieuses comportent deux aspects : la probabilité d'exposition et la sensibilité (c.-à-d. la gravité des conséquences de l'infection). La probabilité d'exposition dépend du nombre d'organismes infectieux ( "l'aléa »), c'est-à-dire les êtres humains infectés, les microorganismes, les vecteurs arthropodes et les hôtes réservoirs d'origine animale, dans notre environnement, et de la fréquence des contacts des êtres humains avec ces organismes. Les changements climatiques devraient favoriser leur augmentation. En même temps, la gravité des conséquences des maladies infectieuses sera probablement plus sévère dans des populations de plus en plus vieillissantes et souffrant de maladies chroniques. Cela semble le cas pour les maladies à transmission vectorielle, comme le virus du Nil occidental (26).

\section{Conclusion}

Le climat du Canada change. En raison de l'augmentation des températures ainsi que de la variabilité spatiale et temporelle des caractéristiques de précipitations, les risques liés à la maladie de Lyme et au virus du Nil occidental, déjà bien présents au Canada, augmenteront probablement, comme les risques de maladies transmises par d'autres tiques ou moustiques et de maladies $d$ 'origine alimentaire. Des renseignements plus détaillés sur les risques, actuels ou futurs, de maladies infectieuses causées par les changements climatiques sont présentés dans les articles de ce numéro. Le risque est probablement aggravé par le fait que le Canada compte une population âgée de plus en plus atteinte de maladies chroniques et chez qui les infections risquent d'être plus graves que chez les jeunes et les personnes en santé. L'identification de ces risques est une activité essentielle pour évaluer la vulnérabilité du pays, et constitue un point de départ pour reconnaître où les efforts en santé publique doivent porter pour réduire la vulnérabilité et l'exposition de la population canadienne.

\section{Déclaration des auteurs}

$\mathrm{NHO}$ et PG ont conçu et coécrit l'article, NHO ayant fourni les éléments relatifs à la santé publique et aux maladies infectieuses, et PG, l'information sur les changements climatiques.

Nicholas Ogden était le rédacteur invité de ce numéro du RMTC, mais il s'est récusé de toute décision rédactionelle concernant ce manuscrit. Les décisions ont été prises par la rédactrice en chef, Patricia Huston, M.D.

\section{Conflit d'intérêts}

Aucun. 


\section{ÉDITORIAL}

\section{Références}

1. Bouchard C, Dibernardo A, Koffi J, Wood H, Leighton PA, Lindsay LR. Augmentation du risque de maladies transmises par les tiques en raison des changements climatiques et environnementaux. Relevé des maladies transmissibles au Canada 2019;45(4):89-98. DOI

2. Ludwig A, Zheng $H$, Vrbova L, Drebot MA, Iranpour $M$, Lindsay LR. Augmentation du risque de maladies endémiques transmises par des moustiques au Canada en raison du changement climatique. Relevé des maladies transmissibles au Canada 2019;45(4):99-107. DOI

3. Ng V, Rees EE, Lindsay RL, Drebot MA, Brownstone T, Sadeghieh T, Khan SU. Les changements climatiques pourraient-ils entraîner la propagation de maladies exotiques transmises par les moustiques au Canada ? Relevé des maladies transmissibles au Canada 2019;45(4):108-18. DOI

4. Smith BA, Fazil A. Quelles seront les répercussions des changements climatiques sur les maladies microbiennes d'origine alimentaire au Canada? Relevé des maladies transmissibles au Canada 2019;45(4):119-25. DOI

5. National Centers for Environmental information. Climate at a glance: global time series. Asheville (NC): NOAA; 2018 Sep. https://www.ncdc.noaa.gov/cag/global/time-series

6. World Meteorological Organization. WMO statement on the state of the global climate in 2017. Geneva (CH): WMO; 2018 (WMO-No. 1212). https://library.wmo.int/doc_num. php?explnum_id=4453

7. Blunden J. Arndt DS, Hartfield G. State of the climate in 2017. Bull Amer Meteor Soc 2018;99(8):Si-S332. DOI

8. Cohen J, Screen JA, Furtado JC, Barlow M, Whittleston D, Coumou D, Francis J, Dethloff K, Entekhabi D, Overland $\mathrm{J}$, Jones J. Recent Arctic amplification and extreme midlatitude weather. Nat Geosci 2014;7(9):627-37. DOI

9. Smith SJ, Edmonds J, Hartin CA, Mundra A, Calvin K. Nearterm acceleration in the rate of temperature change. Nat Clim Chang 2015;5:333-6. DOI

10. Environnement et changement climatique Canada. Données et scenarios climatiques pour le Canada : synthèse des observations et des résultats récents de modélisation. Gatineau (QC): ECCC; 2016 Cat. No.: En84-132/2016F-PDF. http://publications.gc.ca/collections/collection_2016/eccc/ En84-132-2016-fra.pdf

11. Mearns LO, McGinnis S, Korytina D. The NA-CORDEX dataset, version 1.0. NCAR Climate Data Gateway. Boulder (CO): The North American CORDEX Program; 2017 DOI

12. van Vuuren DP, Edmonds J, Kainuma M, Riahi K, Thomson A, Hibbard K, Hurtt GC, Kram T, Krey V, Lamarque JF, Masui T, Meinshausen M, Nakicenovic N, Smith SJ, Rose SK. The representative concentration pathways: an overview. Clim Change 2011;109(1-2):5-31.DOI
13. IPCC. Climate Change 2013: The physical science basis. Contribution of Working Group I to the Fifth Assessment Report of the Intergovernmental Panel on Climate Change. Cambridge (UK); and New York (NY): Cambridge University Press; 2013.

14. Ogden NH, AbdelMalik P, Pulliam JRC. Maladies infectieuses émergentes : prévision et détection. Relevé des maladies transmissibles au Canada. 2017;43(10):232-8. DOI

15. Semenza JC, Lindgren E, Balkanyi L, Espinosa L, Almqvist MS, Penttinen P, Rocklöv J. Determinants and drivers of infectious disease threat events in Europe. Emerg Infect Dis 2016 Apr;22(4):581-9. DOI PubMed

16. Berry P, Clarke KL, Fleury MD, Parker S. Human health. In: Warren FJ, Lemmen DS, editors. Vivre avec les changements climatiques au Canada : perspectives des secteurs relatives aux impacts et à l'adaptation (ON): Gouvernement du Canada; 2014. https://www.rncan.gc.ca/sites/www.nrcan. gc.ca/files/earthsciences/pdf/assess/2014/pdf/Rapportcomplet_Fra.pdf

17. Charron DF, Fleury M, Lindsay LR, Ogden N, Shuster CJ. Santé et changements climatiques : évaluation des vulnérabilitiés et de la capacité d'adaptation au Canada. Chapitre 5: Répercussions des changements climatiques sur les maladies transmises par l'eau, les aliments, les vecteurs et les rongeurs. Ottawa (ON): Santé Canada; 2008. http:// publications.gc.ca/collections/collection_2008/hc-sc/H128-108-528F.pdf

18. Smith KR, Woodward A, Campbell-Lendrum D, Chadee DD, Honda Y, Liu Q, Olwoch JM, Revich B, Sauerborn R. Climate Change 2014: Impacts, Adaptation, and Vulnerability. Part A: Global and Sectoral Aspects. Chapter 11: Human Health: Impacts, Adaptation, and Co-benefits. New York (NY): Cambridge University Press; 2014. pp. 709-54. https://www. ipcc.ch/site/assets/uploads/2018/02/WGIIAR5-Chap11_ FINAL.pdf

19. Romero-Lankao P, Smith JB, Davidson DJ, Diffenbaugh NS, Kinney PL, Kirshen P, Kovacs P, Ruiz LV. Climate Change 2014: Impacts, Adaptation, and Vulnerability. Part B: Regional Aspects. New York (NY): Cambridge University Press; 2014. pp. 1439-98. https://www.ipcc.ch/site/assets/ uploads/2018/02/WGIIAR5-PartB_FINAL.pdf

20. Beard CB, Eisen RJ, Barker CM, Garofalo JF, Hahn M, Hayden M, Monaghan AJ, Ogden NH, Schramm PJ. The impacts of climate change on human health in the United States: a scientific assessment. Chapter 5: Vector-borne diseases. Washington (DC): U.S. Global Change Research Program; 2016. pp. 129-56. DOI

21. Altizer S, Ostfeld RS, Johnson PT, Kutz S, Harvell CD. Climate change and infectious diseases: from evidence to a predictive framework. Science 2013 Aug;341(6145):514-9. DOI PubMed 
22. Cable J, Barber I, Boag B, Ellison AR, Morgan ER, Murray K, Pascoe EL, Sait SM, Wilson AJ, Booth M. Global change, parasite transmission and disease control: lessons from ecology. Philos Trans R Soc Lond B Biol Sci 2017 May;372(1719):20160088. DOI PubMed

23. Ogden $\mathrm{NH}$. Climate change and vector-borne diseases of public health significance. FEMS Microbiol Lett 2017 Oct;364(19): DOl PubMed

24. Ogden NH, Lindsay LR. Effects of climate and climate change on vectors and vector-borne diseases: ticks are different. Trends Parasitol 2016 Aug;32(8):646-56.DOI PubMed

25. Dye C. After 2015: infectious diseases in a new era of health and development. Philos Trans R Soc Lond B Biol Sci 2014 May;369(1645):20130426. DOI PubMed

26. Badawi A, Velummailum R, Ryoo SG, Senthinathan A, Yaghoubi S, Vasileva D, Ostermeier E, Plishka M, Soosaipillai $M$, Arora P. Prevalence of chronic comorbidities in dengue fever and West Nile virus: A systematic review and metaanalysis. PLoS One 2018 Jul;13(7):e0200200. DOI PubMed 